Supplement of Clim. Past, 14, 2071-2087, 2018 https://doi.org/10.5194/cp-14-2071-2018-supplement (C) Author(s) 2018. This work is distributed under the Creative Commons Attribution 4.0 License.

(c) (1)

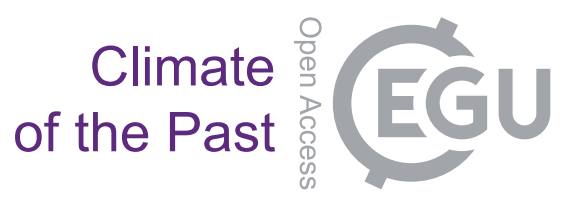

Supplement of

\title{
Climate evolution across the Mid-Brunhes Transition
}

Aaron M. Barth et al.

Correspondence to: Aaron M. Barth (abarth@ehc.edu)

The copyright of individual parts of the supplement might differ from the CC BY 4.0 License. 
- Shakun et al. (2015)

—SST PC1 (this research)
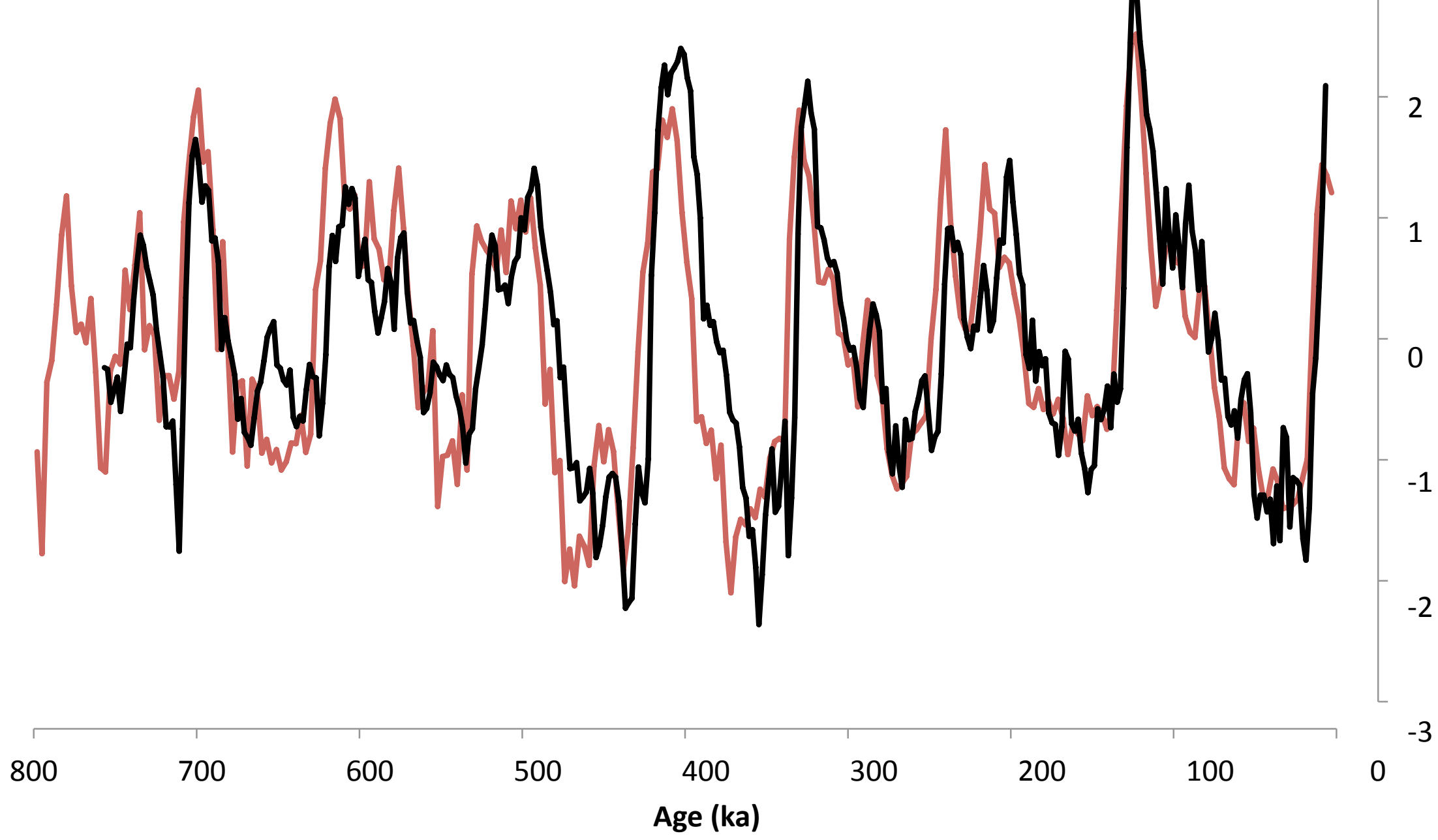

Figure S1 - Comparison of sea-surface temperature results. Results of a sea-surface temperature stack $(\mathrm{n}=49$; red; Shakun et al., 2015) and the first principal component of our sea-surface temperature analysis $(n=15$; black). 


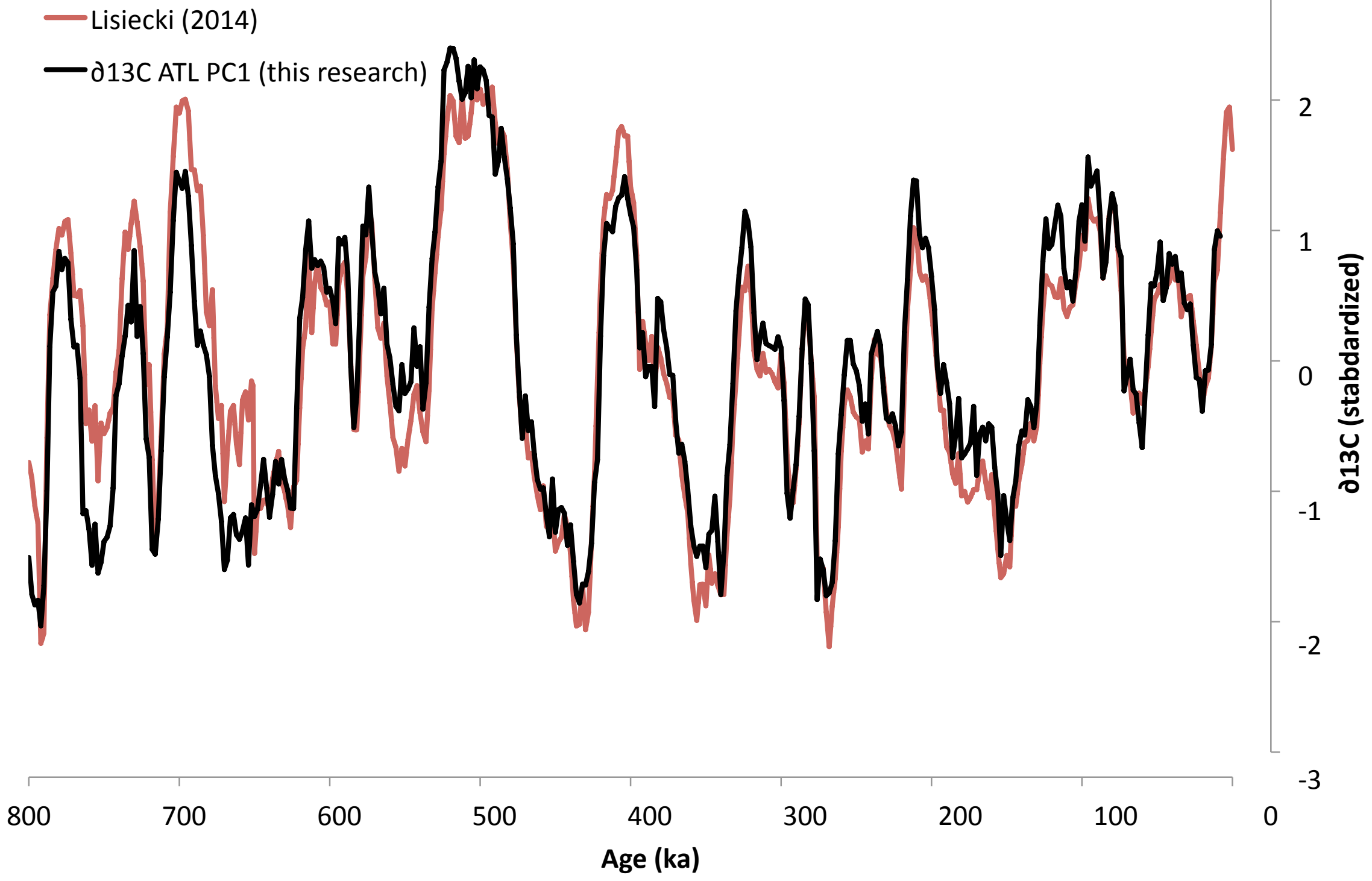

Figure S2 - comparison benthic carbon isotope results. Results of a benthic $\partial^{13} \mathrm{C}$ stack ( $\mathrm{n}=46$; red; Lisiecki, 2014) and the first principal component of our global benthic $\partial^{13} \mathrm{C}$ analysis (black). 


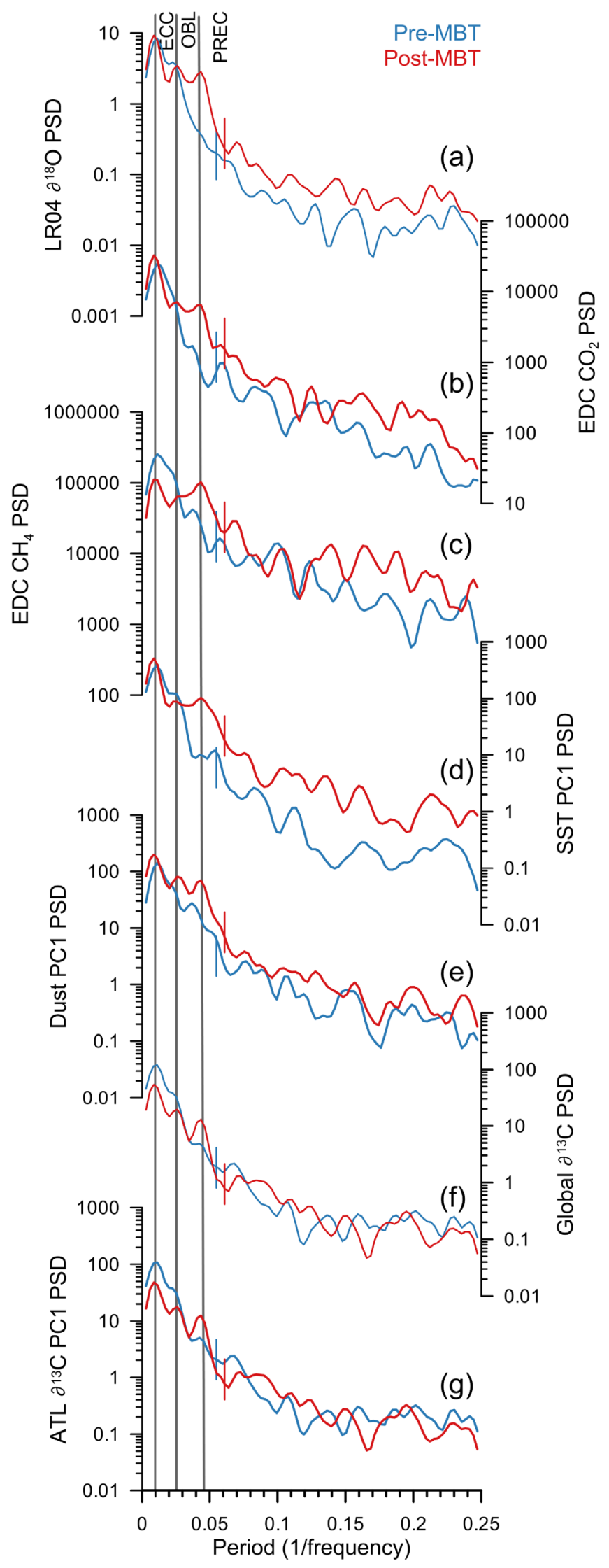

Figure 3 - Spectral power analyses. Power spectral density plots of major climate variables. Blue plot represents the pre-MBT time interval (800-450 ka). Red plot represents the post-MBT (350-8 ka). Vertical lines represent the dominant Milankovitch periods at 100-, 41-, and 23-kyr. a, LR04 benthic oxygen isotope stack. b, EPICA Dome $\mathrm{C} \mathrm{CO}_{2}$. c, EPICA Dome $\mathrm{C} \mathrm{CH}_{4}$. d, Sea-surface temperatures PC1. e, Dust PC1. f, Global $\partial^{13} \mathrm{C} \mathrm{PC1.} \mathrm{g,} \mathrm{Atlantic}$ $\partial^{13} \mathrm{C}$ PC1. 


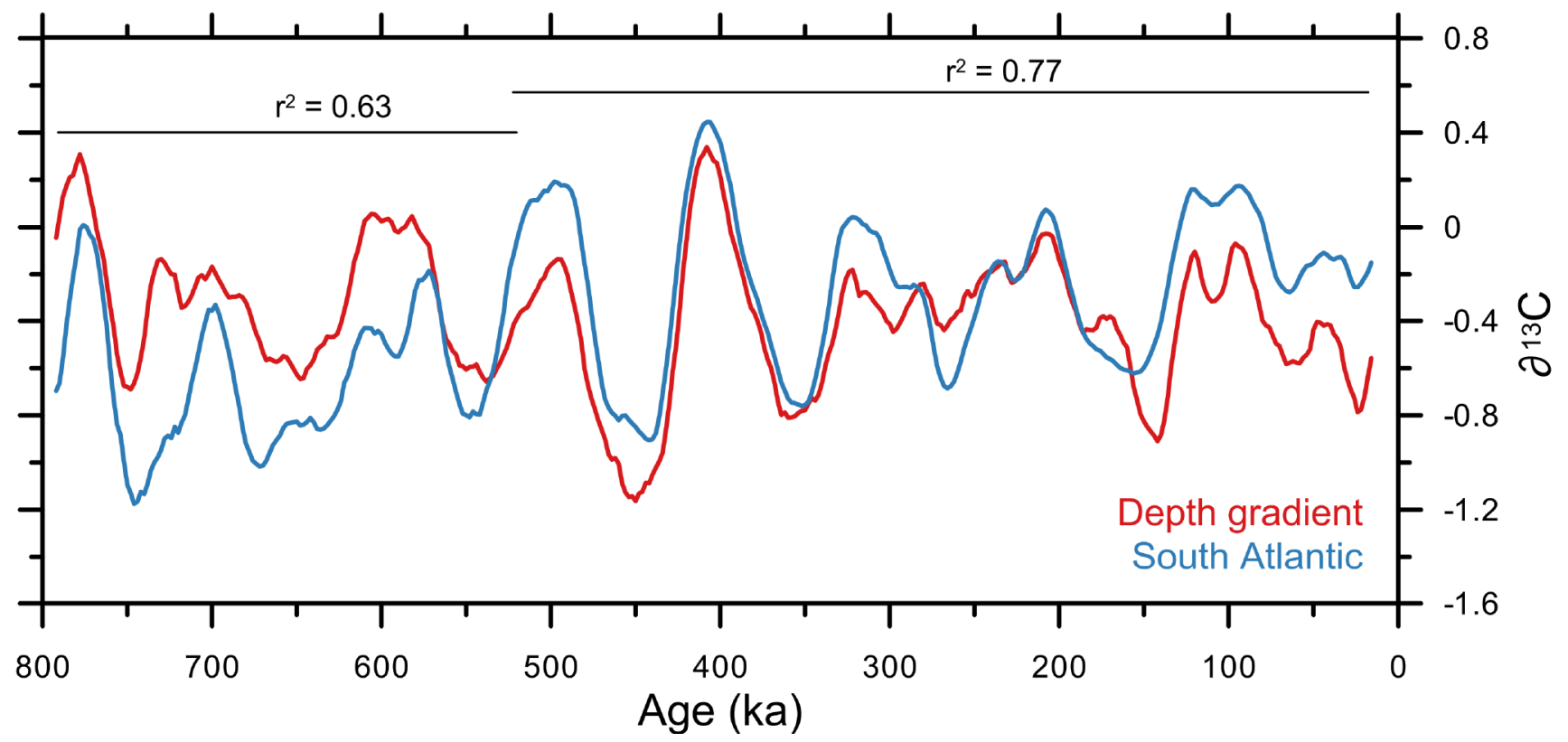

Figure 10 -North Atlantic depth gradient and South Atlantic $\partial^{13} \mathbf{C}$. Depth gradient of the North Atlantic $\partial^{13} \mathrm{C}$ records (Deep minus Intermediate; red) compared with the Deep South Atlantic ${ }^{13} \mathrm{C}$ stack (blue). Each record has been smoothed using a 9-point moving average. Both are plotted in $\partial^{13} \mathrm{C}$ space to highlight the similarity in values once the isotopic influence of the Intermediate North Atlantic is removed. Horizontal lines indicate a period of lower correlation $\left(r^{2}=0.63\right)$ prior to MIS 15 , and higher correlation $\left(r^{2}=0.77\right)$ after the MIS 15 . 


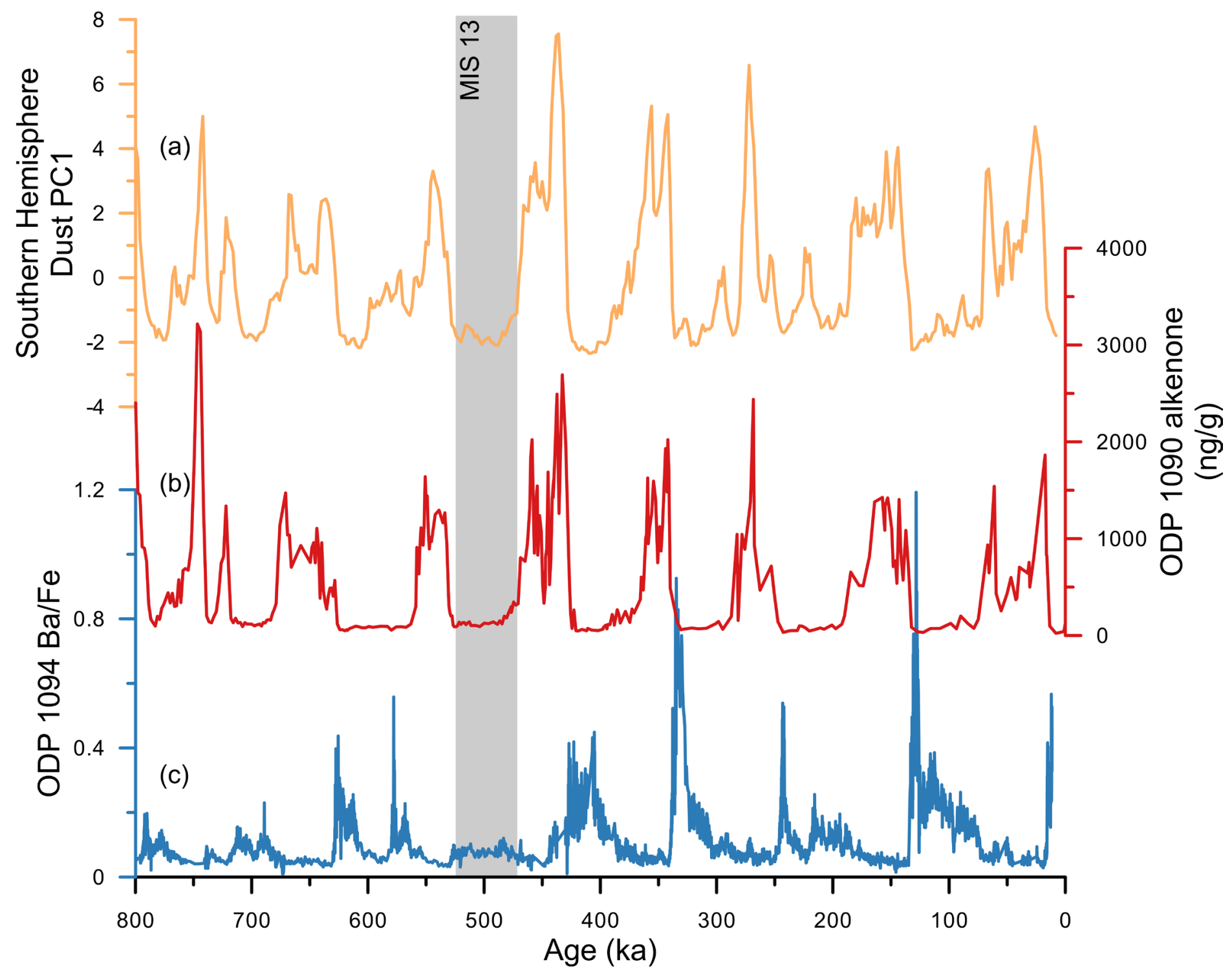

Figure 13 - Dust and productivity. a, the first principal component of southern dust (orange). The y-axis has been inverted to show interglacial periods as up. b, Alkenone record of export productivity from the Subantarctic Zone (ODP 1090; Martinez-Garcia et. al, 2009; red). c, Ba/Fe ratios show export productivity from the Antarctic Zone (ODP 1094; Jaccard et al., 2013; blue). The vertical gray box highlights a period of little to no export productivity or dust during MIS 13. 

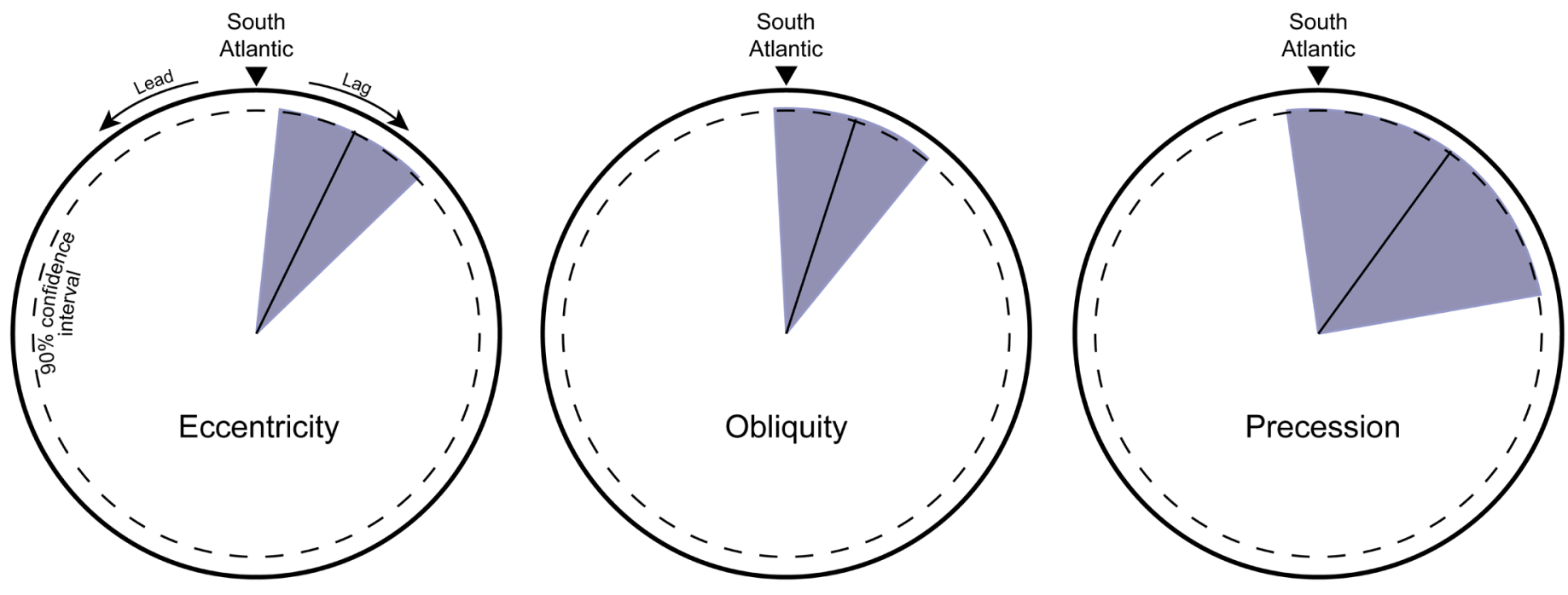

Figure 16 - Post-MBT $\partial^{13} \mathbf{C}$ phase wheels. Phase wheels for each of the Milankovitch cycles (eccentricity, obliquity, and precession) between the North and South Atlantic $\partial^{13} \mathrm{C}$ regional stacks. The arrow at the top of each wheel shows an in-phase relationship of the North Atlantic with the South Atlantic in that frequency band. Values to the right indicate a lag by the North Atlantic relative to the South Atlantic. Values to the left indicate a lead by the North Atlantic relative to the South Atlantic. Purple shading indicates the uncertainty in each relationship. Dotted line highlights the $90 \%$ confidence interval for each frequency. 


\begin{tabular}{|c|c|c|c|c|}
\hline Data Set & Variable & Period & Variance $^{a}$ & F-test ${ }^{b}$ \\
\hline SST_PC1 & SST & Post-MBT & 8.9681 & Reject \\
\hline SST_PC1 & SST & Pre-MBT & 6.5399 & \\
\hline SST_PC2 & SST & Post-MBT & 1.8564 & \\
\hline SST_PC2 & SST & Pre-MBT & 1.2808 & \\
\hline $\mathrm{EDC} \mathrm{CO}{ }_{2}$ & $\mathrm{CO}_{2}$ & Post-MBT & 619.6373 & Reject \\
\hline $\mathrm{EDC} \mathrm{CO}{ }_{2}$ & $\mathrm{CO}_{2}$ & Pre-MBT & 467.1198 & \\
\hline$\partial^{13} \mathrm{C}$ Atlantic PC1 & $\partial^{13} \mathrm{C}$ & Post-MBT & 0.9950 & Reject \\
\hline$\partial^{13} \mathrm{C}$ Atlantic PC1 & $\partial^{13} \mathrm{C}$ & Pre-MBT & 1.9414 & \\
\hline$\partial^{13} \mathrm{C}$ Atlantic PC1 no MIS 13 & $\partial^{13} \mathrm{C}$ & Post-MBT & 0.9950 & Reject \\
\hline$\partial^{13} \mathrm{C}$ Atlantic PC1 no MIS 13 & $\partial^{13} \mathrm{C}$ & Pre-MBT & 1.9414 & \\
\hline$\partial^{13} \mathrm{C}$ Atlantic PC2 & $\partial^{13} \mathrm{C}$ & Post-MBT & 0.2365 & Reject \\
\hline$\partial^{13} \mathrm{C}$ Atlantic PC2 & $\partial^{13} \mathrm{C}$ & Pre-MBT & 0.3378 & \\
\hline$\partial^{13} \mathrm{C}$ Pacific PC1 & $\partial^{13} \mathrm{C}$ & Post-MBT & 0.2283 & Reject \\
\hline$\partial^{13} \mathrm{C}$ Pacific PC1 & $\partial^{13} \mathrm{C}$ & Pre-MBT & 0.4317 & \\
\hline$\partial^{13} \mathrm{C}$ Global & $\partial^{13} \mathrm{C}$ & Post-MBT & 1.1744 & Reject \\
\hline$\partial^{13} \mathrm{C}$ Global & $\partial^{13} \mathrm{C}$ & Pre-MBT & 2.1739 & \\
\hline$\partial^{13} \mathrm{C}$ Global & $\partial^{13} \mathrm{C}$ & Post-MBT & 1.1744 & Reject \\
\hline$\partial^{13} \mathrm{C}$ Global & $\partial^{13} \mathrm{C}$ & Pre-MBT & 2.1739 & \\
\hline LR04 $\partial^{18} \mathrm{O}$ & $\partial^{18} \mathrm{O}$ & Post-MBT & 0.1965 & Reject \\
\hline LR04 $\partial^{18} \mathrm{O}$ & $\partial^{18} \mathrm{O}$ & Pre-MBT & 0.1572 & \\
\hline Dust Global & Dust & Post-MBT & 4.5498 & Reject \\
\hline Dust Global & Dust & Pre-MBT & 2.6819 & \\
\hline $\mathrm{EDC} \mathrm{CH} \mathrm{CH}_{4}$ & $\mathrm{CH}_{4}$ & Post-MBT & 5432.4 & \\
\hline $\mathrm{EDC} \mathrm{CH}_{4}$ & $\mathrm{CH}_{4}$ & Pre-MBT & 6012.4 & \\
\hline EDC Temperature & Deuterium & Post-MBT & 270.1 & Reject \\
\hline EDC Temperature & Deuterium & Pre-MBT & 116.3 & \\
\hline Bottom water temp. & $\mathrm{Mg} / \mathrm{Ca}$ & Post-MBT & 0.0105 & Reject \\
\hline Bottom water temp. & $\mathrm{Mg} / \mathrm{Ca}$ & Pre-MBT & 0.0069 & \\
\hline
\end{tabular}

"Variance values for the intervals "Pre-MBT" (450-800 ka) and "Post-MBT" (0-350 ka)

${ }^{\mathrm{b}} \mathrm{F}$-tests of the null hypothesis that variance in the Pre- and Post-MBT time intervals are the same 
Table S2 - EOF Results

\begin{tabular}{|c|c|c|}
\hline Core & EOF-1 & EOF-2 \\
\hline \multicolumn{3}{|l|}{ Sea-surface temperature } \\
\hline DSDP 607 & 2.4611 & -1.4678 \\
\hline ODP 846 & 0.6214 & 0.1373 \\
\hline ODP 982 & 0.9055 & 0.1431 \\
\hline ODP 1143 & 0.5791 & 0.0256 \\
\hline ODP 1082 & 1.2605 & -0.0162 \\
\hline ODP 1313 & 1.5653 & -0.0557 \\
\hline ODP 722 & 0.6955 & -0.0637 \\
\hline ODP 1146 & 0.6955 & -0.0637 \\
\hline ODP 846 & 0.6214 & 0.1373 \\
\hline MD97-2140 & 0.3085 & 0.0024 \\
\hline MD06-3018 & 0.3711 & 0.1174 \\
\hline ODP 806 & 0.6318 & 0.0738 \\
\hline DSDP 594 & 1.6780 & 2.1688 \\
\hline V22-174 & 0.4994 & -0.0700 \\
\hline RC13-110 & 0.6403 & -0.2426 \\
\hline \multicolumn{3}{|l|}{ Dust } \\
\hline ODP 659 & 0.356 & 0.646 \\
\hline ODP 1090 & 0.9004 & -0.2768 \\
\hline ODP 1090 & 0.8957 & -0.2884 \\
\hline CLP & -0.5902 & -0.4107 \\
\hline PS75-074 & 0.8153 & -0.107 \\
\hline PS75-076 & 0.8914 & -0.0061 \\
\hline ODP 663 & 0.4237 & 0.6842 \\
\hline EPICA Dome C & 0.8729 & -0.1856 \\
\hline \multicolumn{3}{|l|}{ Global $\partial^{13} \mathrm{C}$} \\
\hline ODP 982 & 0.0473 & -0.1403 \\
\hline ODP 983 & 0.1598 & -0.1581 \\
\hline ODP 984 & 0.1412 & -0.4831 \\
\hline DSDP 607 & 0.4186 & 0.0256 \\
\hline U 1308 & 0.3688 & 0.0163 \\
\hline DSDP 502 & 0.0674 & -0.098 \\
\hline ODP 980/981 & 0.2748 & -0.1104 \\
\hline ODP 664 & 0.3818 & 0.0776 \\
\hline ODP 925 & 0.3548 & 0.027 \\
\hline ODP 926 & 0.3949 & 0.0174 \\
\hline ODP 927 & 0.3886 & 0.0161 \\
\hline ODP 929 & 0.4186 & 0.0256 \\
\hline ODP 928 & 0.4069 & 0.0414 \\
\hline ODP 1090 & 0.3774 & 0.1325 \\
\hline ODP 1143 & 0.1641 & -0.0143 \\
\hline ODP 677 & 0.185 & -0.0199 \\
\hline ODP 846 & 0.1795 & -0.0169 \\
\hline ODP 849 & 0.1901 & -0.003 \\
\hline \multicolumn{3}{|l|}{ Atlantic $\partial^{13} \mathrm{C}$} \\
\hline ODP 982 & 0.0459 & 0.1404 \\
\hline ODP 983 & 0.1567 & 0.1579 \\
\hline ODP 984 & 0.1373 & 0.4838 \\
\hline DSDP 607 & 0.423 & -0.0194 \\
\hline U 1308 & 0.3683 & -0.0141 \\
\hline ODP 980/981 & 0.2733 & 0.112 \\
\hline DSDP 502 & 0.0674 & 0.0989 \\
\hline ODP 664 & 0.3805 & -0.0756 \\
\hline ODP 925 & 0.3555 & -0.0242 \\
\hline ODP 926 & 0.3964 & -0.0136 \\
\hline ODP 927 & 0.39 & -0.0129 \\
\hline ODP 929 & 0.423 & -0.0194 \\
\hline ODP 928 & 0.408 & -0.0379 \\
\hline ODP 1090 & 0.3728 & -0.134 \\
\hline \multicolumn{3}{|l|}{ Pacific $\partial^{13} C$} \\
\hline ODP 1143 & 0.5066 & -0.1312 \\
\hline ODP 677 & 0.113 & 0.0389 \\
\hline ODP 846 & 0.1482 & 0.1219 \\
\hline ODP 849 & 0.2123 & 0.2073 \\
\hline
\end{tabular}

